Anna Paul

Nils Altner

«Ich bin ein anderer Mensch geworden», sagt Patientin Anne S., die sich in den Jahren vor ihrem Klinikaufenthalt in Essen nur mit Schmerztabletten über Wasser gehalten hat. «Meine Schmerzen waren wahnsinnig schlimm. Kopfschmerzen, jeden Tag und mit Übelkeit, Gelenkschmerzen an Schulter, Fuss, Knie, Daumen - auf einer Schmerzskala von 0 bis 10 waren meine Schmerzen bei 9. Die Tabletten, die ich nahm, schlugen nicht mehr an.» Sie war am Ende ihrer Kräfte.

Das endemische Auftreten von chronischen Schmerz-, Herz-Kreislauf- und Darmerkrankungen, aber auch die Zunahme von Burnout und Depressionen lassen sich als Zeichen dafür deuten, dass der momentan gesellschaftlich vorherrschende Lebensstil für immer mehr Menschen immer früher in ihrem Leben zu einer Dysbalance zwischen Anforderungen und Bewältigungsressourcen führt. Anstatt Menschen von Kindheit an auf die Befähigung zur Selbstfürsorge und Gesundheitspflege zu orientieren, werden stattdessen meist nur die Symptome behandelt, was den Verbrauch von Analgetika, Antihypertensiva und Psychopharmaka auch mit all ihren Risiken und Nebenwirkungen enorm gesteigert hat. Mangelnde Selbstregulation wird somit durch äussere kostenpflichtige Regulative zu kompensieren versucht. Entfernt sich eine ganze Gesellschaft aber immer weiter von einem Lebensstil, der die inneren Ressourcen für Gesunderhalt und Selbstheilung fördert, wächst das persönliche Leid, die Leistungsfähigkeit und -bereitschaft sinken und der Kollaps des Gesundheitssystems ist vorprogrammiert. Dieser Trend lässt sich nur bremsen, wenn neben die behandelnden Therapien auch partizipative Interventionen treten, die die Patienten zur Selbstfürsorge und zu

\title{
Mind-Body-Medizin bei chronischen Erkrankungen
}

einem nachhaltig gesundheitsfördernden Lebensstil befähigen. Die MindBody-Medizin verfolgt diesen integrativen Ansatz.

So wurde Anne S. 2 Wochen stationär in der Klinik für Naturheilkunde und Integrative Medizin der Kliniken Essen-Mitte behandelt. Unter Anleitung des Pflegepersonals führte sie regelmässig selbstständig ein Kopfschmerzenstufenschema mit Euminz-Stift, Lidocain-Nasenspray und Weidenrinde-Tabletten durch. Zusätzlich bekam sie die Schabmassage Gua Sha und Akupunktur. Während des stationären Aufenthalts wurden ernährungsphysiologische Aspekte zur mediterranen Vollwertkost in Theorie und Praxis vermittelt. Anne S. lernte - ebenfalls theoretisch und praktisch - die gesundheitsfördernden Aspekte von Bewegung kennen. Sie führte ein moderates Ausdauertraining in Form von Walking durch und erlernte Qigong-Übungen. Ausserdem erhielt sie lokale Wärmeanwendungen in Form von ZappSackAuflagen (einer durchblutungssteigernden Ingwer-Getreide-Auflage), Senfmehl-Fussbäder und Bienenwachsauflagen sowie als vegetativ umstimmende Massnahmen Kneippsche Brustwickel.

Mind-body-medizinisch wurde ausserdem in den Bereichen Entspannung und Stressregulation eine Lebensstilmodifikation angeregt. Dazu gehörte im Sinne einer kognitiven Umstrukturierung auch eine Reflexion ihres persönlichen Umgangs mit der Erkrankung. Es wurde ihr das Entspannungsverfahren der progressiven Muskelentspannung vermittelt, ausserdem Visualisierungsübungen und Methoden der Achtsamkeitsmeditation.

Vertiefen konnte Anne S. die Themen im Anschluss an den stationären
Aufenthalt in der Tagesklinik, die über 10 Wochen hinweg einmal wöchentlich mit $6 \mathrm{~h}$ in einer festen Gruppe angeboten wird. Ziel war es dort, eine achtsame Haltung zu kultivieren und die als heilsam erlebten Lebensstilveränderungen mithilfe regelmässiger Übungen, die es zu Hause durchzuführen galt, tatsächlich in den Alltag $\mathrm{zu}$ integrieren. Es ging auch darum, die Wahrnehmung für ihre Belastungsgrenzen zu schulen und damit eine respektierende Selbstfürsorge zu praktizieren. Die Gruppe unterstützte sie hier sozial ebenso wie emotional und die Teilnehmer motivierten einander gegenseitig durch vertrauensvollen Erfahrungsaustausch.

«Das Krankenhaus hat mir den Körper wieder gegeben - in der Tagesklinik ist dann meine Seele wieder bei mir angekommen», so Anne S. heute; mittlerweile schmerzfrei. «Ich arbeite natürlich weiter an mir», betont sie, denn das sei entscheidend für ihr Wohlergehen. «Diese Krankheit ist das Beste, was mir in meinem Leben passieren konnte, weil ich ganz viel gelernt habe. Ich habe gelernt, mich zu entspannen. Ich habe gelernt, vieles anders zu sehen. Es geht mir einfach richtig gut.»

Die Klinik und der Alfried Krupp von Bohlen und Halbach-Stiftungslehrstuhl für Naturheilkunde der Universität Duisburg-Essen engagiert sich in der Aus- und Weiterbildung. So findet vom 23. bis 26. August 2012 eine Mind/Body Medicine Summer School statt. Ausserdem werden Vertiefungsseminare angeboten, in denen Mind-Body-Interventionen erlernt werden können. Beide Angebote sind von der deutschen Ärztekammer als Fortbildung anerkannt und Fortbildungspunkte sind beantragt. Näheres unter www.mindbodymedicine.de.
Dr. rer. medic. Anna Paul

Leitung Ordnungstherapie, Mind-Body-Medicine

Naturheilkunde und Integrative Medizin

Kliniken Essen-Mitte, Knappschafts-Krankenhaus

Am Deimelsberg 34a, 45276 Essen, Deutschland

Tel. +49 201 174-25501, Fax -25000; a.paul@kliniken-essen-mitte.de 